\title{
Extramural Venous Invasion in Rectal Cancer: Relationship between Magnetic Resonance Imaging Demonstration and Histopathological Results
}

\author{
Ana Rita Gomes ${ }^{1}$, Carlos Costa Pereira ${ }^{2}$, Olga Oliveira ${ }^{2}$, Fernanda Nogueira², Nuno Oliveira ${ }^{2}$, \\ Joaquim Costa Pereira ${ }^{2}$, Catarina Costa ${ }^{3}$, Sandra F. Martins ${ }^{1,2,4^{*}}$
}

Corresponding author:

Sandra F. Martins, PhD

Life and Health Sciences Research Institute (ICVS), School of Medicine University of Minho

Campus de Gualtar, 4710-057 Braga

Portugal

Phone: +351253604828

FAX: + 351253604820

E-mail: sandramartins@med.uminho.pt
'School of Medicine, University of Minho, Braga, Portugal

${ }^{2}$ Surgery Department, Coloproctology Unit, Braga Hospital, Braga, Portugal

${ }^{3}$ Radiology Department, Braga Hospital, Braga, Portugal

4ICVS/3B's - PT Government Associate Laboratory, Braga/Guimarăes, Portugal

\section{ABSTRACT}

Background: Preoperative staging of rectal cancer $(\mathrm{RC})$ with magnetic resonance imaging (MRI) made it possible to individualize treatment and select high-risk patients who will benefit from neoadjuvant treatment, by identifying prognostic risk factors, which were previously established only through histopathological studies. Among these prognostic risk factors, emphasis should be given to extramural venous invasion (EMVI) and extramural depth of tumor (EMDT).

Methods: This study aimed to evaluate the following issues: first the accuracy of MRI for detection of EMVI compared to histopathologic analysis second to evaluate the prognostic value of EMVI determined by MRI and third to establish the relationship between EMVI and EMDT.

Results: MRI staging of 151 patients that underwent surgical resection for $\mathrm{RC}$, were analyzed for MRI grading of EMVI, using a score of three levels. Comparison between MRI grading of EMVI and histopathology made it possible to conclude that there was no significant difference between the two methods. As far as the prognostic value of EMVI, the estimated average survival rate and estimated relapse-free survival were higher in patients with degree 0 . Furthermore, there was a strong positive correlation between EMVI and EMDT from MRI. Conclusions: MRI grading of EMVI correlates with histopathological findings and should be included in the multidisciplinary preoperative discussion of RC patients.

Key words: rectal cancer, extramural venous invasion, extramural depth of tumor

\section{INTRODUCTION}

Received: 13.11 .2019
Colorectal cancer (CRC) is the third most diagnosed cancer worldwide. In 2012, CRC was ranked third among cancer incidence with a total of 1.4 million patients (9.7\% of the total) (1).

Tumor location influences the prognosis of CRC. Rectal cancer (RC) occurs in 
one third of CRC cases and has the worst prognosis, due to the higher metastasis frequency at diagnosis and the highest local relapse rate (2). In the last two decades, the treatment of patients with RC has improved $(3,4)$. Nowadays, patients with higher tumor stages are treated with neoadjuvant chemoradiotherapy to achieve a lower local relapse rate (5). Radiotherapy improved survival $(6,7)$ and magnetic resonance imaging (MRI) offers an improvement in staging. MRI results individualize treatment and allowed the selection of high-risk patients who will benefit from neoadjuvant treatment $(2,8-10)$, by identifying prognostic risk factors which were previously established only through histopathological studies, namely: circumferential resection margin (CRM), lymph node involvement, extramural venous invasion (EMVI) and extramural depth of tumor (EMDT) (9-12).

The presence of malignant cells in the vasculature of the mesorectum is called EMVI and was proven to be an independent histologic predictor of disease relapse and poorer overall survival (13-14). According the results of the MERCURY (Magnetic Resonance Imaging and Rectal Cancer European Equivalence Study) Study Group EMVI can be detected by MRI and its severity is associated with relapse's brevity $(15,16)$. Using MRI for staging, one of the following criteria would be suggestive for EMVI: pattern of tumor margin, location of tumor relative to vessels, vessel caliber and vessel border (17). As tumor invades along the lumen, the vessel expands, the vessel caliber increases and may acquire an intermediate intensity signal similar to the tumor's (17).

The importance of EMVI assessment has increased so far, but has not already being considered in the main American guidelines. Conversely the EMVI detection by MRI is supported and recommended by the MERCURY Study Group in order to determine the usefulness of neoadjuvant treatment $(18,19)$.

EMDT corresponds to the tumor extension in the mesorectum and is a histologic predictor of poor prognosis and can be measured by MRI (11).

This study aimed to evaluate the accuracy of MRI for detection of EMVI compared to histopathologic analysis, to evaluate the prognostic value of EMVI determined by MRI and to establish the relationship between EMVI and EMDT.

\section{METHODS}

Between January 2007 and June 2015222 patients with RC (adenocarcinoma) underwent surgical resection in curative intent,at Braga's Hospital and were included for this study. Exclusion criteria were defined as follows: patients with a histological diagnosis differing from the above; patients who did not undergo surgical treatment; patients who didn't undergo MRI prior to surgery or missing MRI files; patients without results from pathology staging; patients with Familial Adenomatous Polyposis or Lynch Syndrome.

Clinical and staging data included: age, gender, tumor location, neoadjuvant treatment and T/N staging by means of MRI. Histological tumor analyses: T/N staging, presence of venous and lymphatic invasion. Follow-up/outcomes: survival and disease-free survival (DFS), defined as the period between diagnosis and relapse(local or distant), in months.

New parameters were assessed through MRI, namely EMVI and EMDT.

\section{Methodology used for MRI interpretation}

Following the protocol utilized by the MERCURY Study Group, for EMVI and EMDT assessment, T2-weighted sequences perpendicular to the long axis of the rectum were used (11). Whenever possible, and as recommended, $3 \mathrm{~mm}$ section thickness sequences were used.

MRI images were reviewed by an expert radiologist, who has more than 7 years of experience in interpreting rectal MRI images, blinded to the results of histopathology.

\section{EMVI assessment}

The following EMVI suggestive features were considered (17): tumor with nodular/irregular margins; tumor or tumor deposits adjacent to vessels; Vessels with an intermediate intensity signal similar to the tumors; Vessels with increased caliber; and Vessels with nodular/irregular borders. According to the presence or absence of such features, the following score was defined: Score 0 (absent): none of the above features were detected; Score 1 (suspicion): one or more of the above features were detected, with the exception of caliber or border vessel's changes and Score 2 (evident): detection of, at least, vessels with an increased caliber and nodular/irregular borders.

\section{EMDT assessment}

EMDT, defined as the extension of $\mathrm{T} 3$ and $\mathrm{T} 4$ tumor that invades the mesorectum, was measured in $\mathrm{mm}$ and categorized according to the following: inferior or equal $(\leq)$ to 5 or higher $(>)$ than 5 . 


\section{Statistical analysis}

Descriptive analysis of the variables under study was performed providing frequencies, means, standard deviations (SD), medians and interquartile range.

Statistical analysis was performed with Statistical Package for Social Sciences (SPSS) version 22.0 statistical software. Categorical data were presented by absolute and relative frequencies (percentage) and compared using Pearson's $\chi^{2}$ test or Fisher's exact test. Quantitative data were summarized as mean (standard deviation) or median (interquartile range) and compared depending on the pattern of distribution using Student's t-test or Wilcoxon test.

Acceptable range for normality of the quantitative variables is skewness and kurtosis lying between -1 to 1 . Aside from EMDT, all quantitative variables were normally distributed.

For calculating sensitivity, specificity, positive and negative predictive values (PV) of EMVI assessment through MRI, the online tool MedCalc ${ }^{\circledR}$ (available in http://www.medcalc.org/calc/diagnostic test.php) was used.

In order to understand whether the proportion of cases in which MRI detected EMVI differs from the proportion given by histopathologic analysis, the McNemar test was used. For the assessment of the survival and DFS outcomes, a Kaplan-Meier analysis was performed. Spearman correlation (rho) was used to establish the relationship between EMVI and EMDT.

For all tests, it was assumed a significance of 0.05 and a confidence interval $(\mathrm{Cl})$ of $95 \%$.

\section{Ethics approval and consent to participate}

This project was approved by Braga Hospital Ethics Committee (CESHB 123/2015) and also by Ethics Subcommittee for Life and Health Sciences. The study was conducted in compliance with the Declaration of Helsinki. All research was performed in accordance with relevant guidelines and regulations; informed consent was obtained from all participants and/or their legal guardians.

\section{RESULTS}

\section{Sample characterization}

The study sample consists of 151 patients who met inclusion criteria. Of these, $61.6 \%(n=93)$ were male and $38.4 \%(n=58)$ female, aged between 29 and 90 years (mean=67.2 and $S D=12.9$ ).
In $62.9 \%(n=95)$ of the cases the tumor was located in the mid-rectum.

Regarding MRI tumor staging, 34.4\% ( $n=52)$ of the tumors were classified as T2 and $58.9 \%(n=89)$ as T3, and in $53 \%(n=80)$ of patients a positive lymph node involvement occurred.

According to assessment of EMVI by MRI, 52.3\% $(n=79)$ were classified as score $0,36.4 \%(n=55)$ as score 1 and $11.3 \%(n=12)$ as score 2 . Histopathologic analysis revealed in $81.5 \%(n=123)$ no EMVI.

Table 1 gives an overview of the radiological and pathological characteristics.

The proportion of staged T3/T4 tumors and lymph node involvement detected by MRI were more frequent in the group with EMVI score 1 and $2-84.4 \%$, $\mathrm{n}=61$ and $66.7 \%, \mathrm{n}=48$, respectively - as shown in fig. 1 .

Nearly $41 \%(n=62)$ of the patients did not receive neoadjuvant treatment and $49 \%(n=74)$ were submitted to preoperative concurrent chemoradiotherapy. In August 2017, 18.5\% ( $n=28)$ of the patients had a recurrence (local or distant) and $23.8 \%(n=36)$ died.

\section{Comparison of MRI grading of EMVI with histopathology}

Among the 123 cases with missing EMVI in histopathology, 54.5\% ( $n=67)$ had also MRI-EMVI score 0 . However, in $36.6 \%(n=45)$ of the cases, assessment

Table 1 -MRI and histopathological tumor characterization

\begin{tabular}{cc}
\hline Patient characteristics & $\mathbf{n ~ 1 5 1 ( \% )}$ \\
\hline Tumor localization & $31(20.5)$ \\
Low-rectum & $95(62.9)$ \\
Mid-rectum & $25(16.6)$ \\
High-rectum & \\
\hline MRI - T stage & $3(2)$ \\
T1 & $52(34.4)$ \\
T2 & $89(58.9)$ \\
T3 & $7(4.6)$ \\
T4 & \\
\hline MRI - N status & $80(53)$ \\
N+ & $71(47)$ \\
N0 & $79(52.3)$ \\
\hline MRI - EMVI score & $55(36.4)$ \\
Score 0 & $17(11.3)$ \\
Score 1 & \\
Score 2 & $19(12.6)$ \\
\hline Histopathological venous vascular invasion & $123(81.5)$ \\
Present & $9(6)$ \\
No evidence & \\
Not reported & $28(18.5)$ \\
\hline Histopathologicallymphatic vascular invasion & $109(72.2)$ \\
Present & $14(9.3)$ \\
No evidence & \\
Not reported & \\
\hline T= tumor; N= nodal; EMVI=Extramural Venous Invasion & \\
\hline
\end{tabular}


Figure 1 - Frequency of $\mathrm{T} / \mathrm{N}$ staging by EMVI score, by means of MRI:

(i) in the group of patients with EMVI score 0 , the following cases were identified: 44 of T1/T2; 35 of T3/T4; 47 without lymph node involvement (NO) and 32 with lymph node involvement $(\mathrm{N}+)$; (ii) in the group of patients with EMVI score 1 or 2, the following cases were identified: 11 of T1/T2; 61 of T3/T4; 24 NO and $48 \mathrm{~N}+$

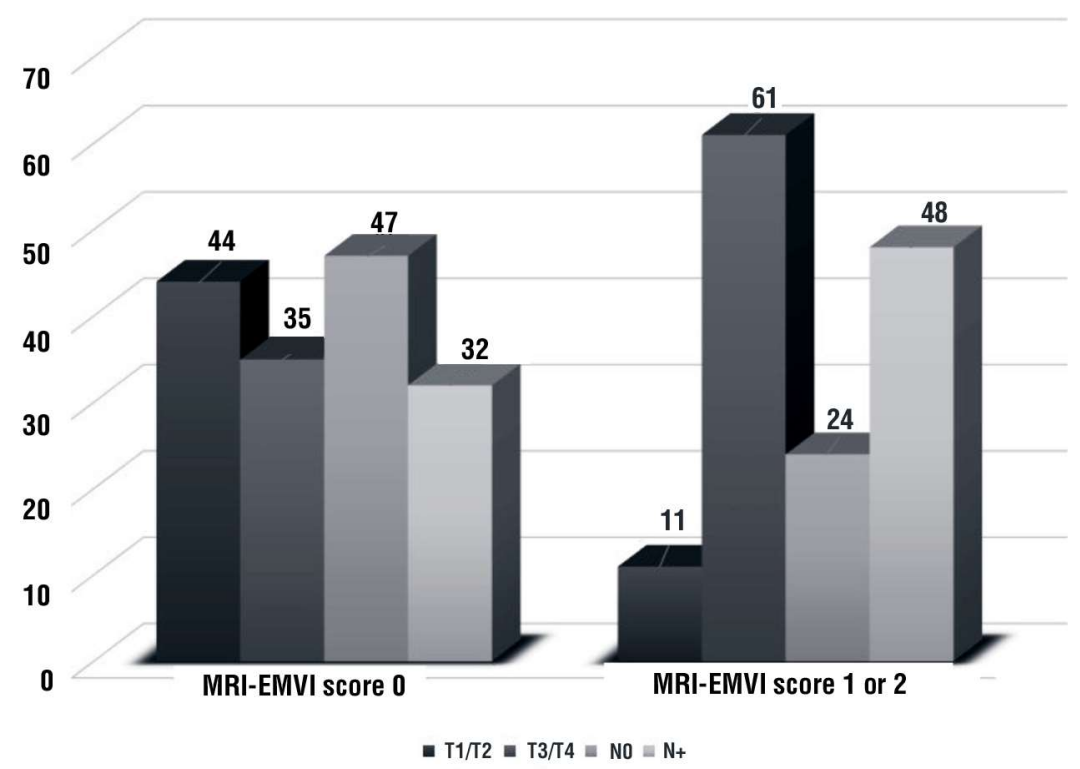

through MRI did not exclude venous invasion, hence being classified as score 1 , and in $8.9 \%(n=11)$ of the cases EMVI as score 2. Among the 19 cases with evidence of EMVI in histopathology, 26.3\% ( $n=5)$ were classified as MRI-EMVI score 1 and $26.3 \%(n=5)$ as MRI-EMVI score 2.

Thus, in $47.2 \%$ ( $n=67)$ of the cases both methods of diagnosis (histopathology and MRI) are in accordance with the absence of EMVI, whereas in $7 \%(n=10)$ of the cases both methods point out the presence of EMVI. In the 9 cases where a statement regarding EMVI is missing in the histopathological report, MRI assessment leads to the following results: 3 with MRI-EMVI score 0; 5 with MRI-EMVI score 1 and 1 with MRI-EMVI score 2 . The results are as described in table 2.

In comparison between MRI-EMVI score and histopathology we could not overserve a statistically significant difference between the two methods of EMVI diagnosis, using the EMVI score 0 or 2 for all patients $(p=.824)$ and only considering without neoadjuvant treatment $(p=.375)$. The difference between the proportion of cases in which MRI detected EMVI and the proportion given by histopathologic analysis isn't statistically significant.

Tables 3 summarize the sensitivity, specificity, positive predictive values (PPV) and negative predictive values (NPV) of EMVI assessment by MRI.

\section{Survival and DFS according to MRI-EMVI score}

According to Kaplan Meier analysis, the mean survival rate in the group of MRI-EMVI score 0 was 68.9 months ( $\mathrm{SE}=4.2,95 \% \mathrm{Cl} 60.7$ - 77.2), respectively 63.6 months ( $\mathrm{SE}=4,95 \% \mathrm{Cl} 55.9$ - 71.4) in the group with score 1 or 2 . The difference between the two groups was not significant (Log Rank: $p=.461$ ) - as shown in fig. 2.
Table 2 - Frequency of EMVI assessment at MRI and histopathology

\begin{tabular}{|c|c|c|c|c|}
\hline \multicolumn{5}{|c|}{ MRI-EMVI score } \\
\hline & Score 0 & Score 1 & Score 2 & $\mathrm{n}=151$ patients \\
\hline No evidence & $\begin{array}{c}67 \\
(54.5) \\
(47.2)\end{array}$ & $\begin{array}{c}45 \\
(36.6) \\
(31.7)\end{array}$ & $\begin{array}{c}11 \\
(8.9) \\
(7.7)\end{array}$ & $\begin{array}{c}123 \text { patients } \\
\% \text { of Histopathology } \\
\% \text { of Total }\end{array}$ \\
\hline Present & $\begin{array}{c}9 \\
(47.4) \\
(6.3)\end{array}$ & $\begin{array}{c}5 \\
(26.3) \\
(3.5)\end{array}$ & $\begin{array}{c}5 \\
(26.3) \\
(3.5)\end{array}$ & $\begin{array}{c}19 \text { patients } \\
\% \text { of Histopathology } \\
\% \text { of Total }\end{array}$ \\
\hline Not reported & $\begin{array}{c}3 \\
(33.3) \\
(53.5)\end{array}$ & $\begin{array}{c}5 \\
(55.6) \\
(35.2)\end{array}$ & $\begin{array}{c}1 \\
(11.1) \\
(11.3)\end{array}$ & $\begin{array}{c}9 \text { patients } \\
\% \text { of Histopathology } \\
\% \text { of Total }\end{array}$ \\
\hline
\end{tabular}

EMVI - Extramural Venous Invasion 
Table 3 - The sensitivity, specificity, PPV and NPV of MRI-EMVI score, in all patients and those with neoadjuvant therapy

\begin{tabular}{|c|c|c|c|c|}
\hline EMVI score & $\begin{array}{l}\text { Sensitivity (\%) } \\
(95 \% \mathrm{CI})\end{array}$ & $\begin{array}{c}\text { Specificity (\%) } \\
\text { (95\% Cl) }\end{array}$ & $\begin{array}{l}\text { PPV (\%) } \\
(95 \% \text { CI) }\end{array}$ & $\begin{array}{l}\text { NPV (\%) } \\
(95 \% \text { CI) }\end{array}$ \\
\hline 0,1 and 2 & $\begin{array}{c}52.6 \\
(28.9-75.6)\end{array}$ & $\begin{array}{c}54.5 \\
(45.3-63.5)\end{array}$ & $\begin{array}{c}15.2 \\
(7.5-26.1)\end{array}$ & $\begin{array}{c}88.2 \\
(78.7-94.4)\end{array}$ \\
\hline 0 and 2 & $\begin{array}{c}35.7 \\
(12.8-64.9)\end{array}$ & $\begin{array}{c}85.9 \\
(76.2-92.7)\end{array}$ & $\begin{array}{c}31.3 \\
(11.0-58.7)\end{array}$ & $\begin{array}{c}88.2 \\
(78.7-94.4)\end{array}$ \\
\hline 0,1 and 2 & $\begin{array}{c}50 \\
(15.7-84.3)\end{array}$ & $\begin{array}{c}70 \\
(55.6-82.1)\end{array}$ & $\begin{array}{c}21.1 \\
(6.1-45.6)\end{array}$ & $\begin{array}{c}89.7 \\
(75.9-97.1)\end{array}$ \\
\hline 0 and 2 & $\begin{array}{c}20 \\
(0.5-71.6)\end{array}$ & $\begin{array}{c}97.22 \\
(85.5-99.9)\end{array}$ & $\begin{array}{c}50 \\
(1.3-98.7)\end{array}$ & $\begin{array}{c}89.74 \\
(75.8-97.1)\end{array}$ \\
\hline
\end{tabular}

The mean DFS in the group of MRI-EMVI score Owas 73.6 months (SE $=3.8,95 \% \mathrm{Cl} 66.3$ - 81), and 71.1 months ( $\mathrm{SE}=4.5,95 \% \mathrm{Cl} 62.8-79.5$ ) in the group with score 1 or 2 . The difference between the two groups was not significant (Log Rank: $p=.922$ ) - as shown in fig. 3.

\section{The relationship between EMVI and EMDT measurements at MRI}

EMDT ranged from 0 to $55 \mathrm{~mm}$ (mean $=4$, interquartile range $=7$ ). Among the 98 patients (64.9\%) with EDMT $\leq 5 \mathrm{~mm}, 60.2 \%(\mathrm{n}=59)$ had MRIEMVI score 0. Among the 53 patients (35.1\%) with EMDT $>5 \mathrm{~mm}, 62.3 \%$ had MRI-EMVI score $1(n=23)$ or score $2(n=10)$.

A strong positive correlation (rho $=.64, p<.001$ ) between EMDT and EMVI was observed.

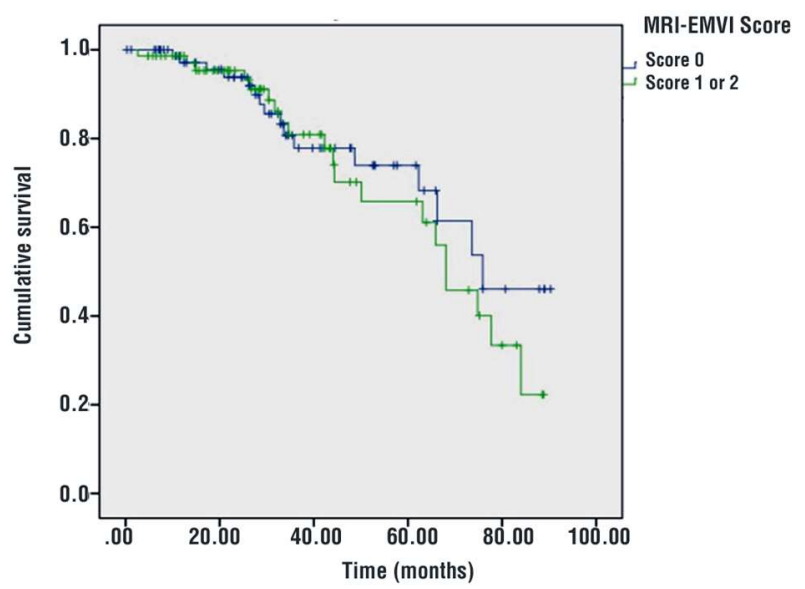

Figure 2 - Kapan Meier survival curves depending of the absence (grade 0 ) or presence (grades 1 and 2) of IVE in MRI. There is a tendency for a lower survival in patients with venous involvement in MRI, after $\mathbf{4 0}$ months of diagnosis. The proportion of survival at 5 years is around $68.8 \%$ in patients with grade 0 of IVE and $65 \%$ in patients with grade 1 or 2 of IVE.

\section{MRI technical parameters}

The quality of MRI images, defined according to the protocol utilized by the MERCURY Study Group (see Material and Methods), was completed in $44.4 \%$ of the cases.

\section{DISCUSSION}

The identification of prognostic risk factors for patients with RC by histopathology provides important information about survival probability or risk of disease recurrence. After resection the histopathological results offers the opportunity to predict to statistical probability of OS and DFS. A preoperative risk stratification of patients with possible tumor remission by neoadjuvant treatment prior to surgery would be desirable (9).

The introduction of neoadjuvant treatment and the

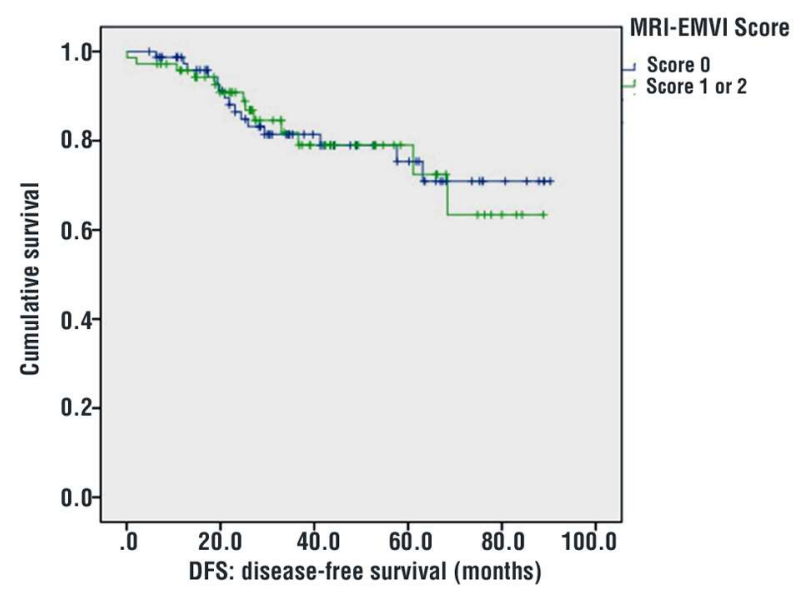

Figure 3 - Kapan Meierdisease survival curvesdepending of the absence (grade 0 ) or presence (grades 1 and 2) of IVE in MRI. The proportion of 5-year relapse-free patients is around $77 \%$ in patients with grade 0 of IVE and $72 \%$ in patients with grade 1 or 2 of IVE. 
total mesorectal excision improved surgical long-term outcomes, reduced local relapse and increased survival rates $(5,20)$. These outcomes attest the importance of using a staging method, which allows to identify prognostic risk factors prior to surgery, thus permitting to adjust treatment to each patient (9).

MRI has become an indispensable tool in staging of $\mathrm{RC}$, revealing a higher accuracy than that of other methods of diagnosis, e.g. computerized axial tomography or endoluminal ultrasound $(9,21)$. Besides, the accuracy and reproducibility of $\mathrm{MRI}$ in identifying prognostic factors, like EMDT and CRM, prior to surgery, is currently accepted $(11,12)$.

Despite the majority of the previous studies having focused on the two parameters mentioned above, a recent study, carried out by Smith et al., concluded that high resolution MRI (T2-weighted MRI with $3 \mathrm{~mm}$ sections) may be used to detect EMVI, with prognostic value (16). Based on the histological reference standard, it demonstrated that the sensitivity and specificity of MRI detection of EMVI in 94 patients, undergoing primary surgery, were $62 \%$ and $88 \%$ respectively (16). For the comparison with histopathological results, the authors proposed a MRI-EMVI score ranging from 0 to 4 . Scores 3 and 4 correspond to a positive EMVI and a score of 0 to 2 correspond to a negative EMVI.

In the present study, considering the group of patients not receiving neoadjuvant treatment as a potential confounder of results, we could observe no significant differences $(p=.375)$, between EMVI at MRI and at histopathology, omitting MRI-EMVI score 1. In fact, in 17 patients, with MRI-EMVI score 1 (suspicious), the venous involvement was confirmed by histopathology in only 3 cases. These conclusions are in line with those of Smith et al., where low MRI-EMVI scores aren't associated with the presence of EMVI at histopathology $(16,17)$.

Ignoring MRI-EMVI score 1 and concerning the group of patients without neoadjuvant treatment, the following results were observed: sensitivity of $20 \%(95 \% \mathrm{Cl}, 0.51-71.64)$, specificity of $97 \%(95 \% \mathrm{Cl}$, 85.47-99.93), PPV (Preditive Positive value) of 50\% $(95 \% \mathrm{Cl}, 1.26-98.74)$ and a NPV (Negative Preditive Value) of $90 \%$ (95\% Cl, 75.78-97.13). In the present study, MRI-EMVI score 2 corresponds to Smith et al.'s MRI-EMVI scores 3 and 4 . Therefore it is possible to compare results between the two studies. Lower sensitivity but higher specificity, were obtained. These sensitivity results may be due to the lower number of individuals in this group. However, sensitivityto detect EMVI at MRI increases when considering the complete score (0, 1 and 2 scores), in patients with $(52.6 \% ; 95 \% \mathrm{Cl}, 28.9-75.6)$ and without neoadjuvant treatment $(50 \% ; 95 \% \mathrm{Cl}, 15.7$ 84.3).

As for high specificity and NPV found, it is possible to conclude that MRI-EMVI score 0 excludes, with certainty, venous involvement. If MRI-EMVI is considered to be used as a criteria for neoadjuvant treatment, as suggested by the MERCURY Study Group, its exclusion by MRI staging may be crucial for therapy decisions and diminish the risk of under treatment.

It is also essential to compare the advantages and limitations of these two diagnostic methods. Microscopic EMVI cannot be detected by MRI, because of resolution limitations (17). However, since patients with large-vessels invasion have the worst outcomes, the clinical impact of EMVI of smallvessels not detected by MRI may be minimal $(17,22)$. Concerning the involvement of the greater caliber vessels, Koh et al. revealed that MRI has $100 \%$ sensitivity and $89 \%$ specificity in identifying EMVI in veins with diameter of at least $3 \mathrm{~mm} \mathrm{10}$. Another advantage of thin-sections T2-weighted MRI images is the possibility of assessing the vessels' course and its positional relation with the tumor, by using image sequences (17).

Based on the incidence variability of EMVI reported in literature (ranges from $8 \%$ to $81 \%$ ) detected by histological studies Messenger et al. suggested that venous invasion has been widely underreported due to the fact of limitations of histopathological examination (23). These authors believe that a possible reason for underestimation of EMVI in histopathological examination is the fact a non-consistent method for discrimination between the venous vessels and the lymphatic vessels (23). Thus, it can't be ruled out that, in the present study, cases of MRI-EMVI score 1 or 2 have been wrongly classified at histopathology as lymphatic invasion instead of venous invasion. Furthermore, extensive vascular invasion can destroy the vessel wall, leaving little evidence of normal vessel wall architecture, which may result in challenging pathological interpretation (17).

Our study has some limitations. Considering the above-mentioned limitations, it is pertinent to propose a revision of the pathological findings of patients without histological EMVI involvement with MRI-EMVI score 1 or 2. Additionally this should be performed in patients where the histological examination does not report the presence or absence of EMVI, and MRI-EMVI score $1(n=5)$ or $2(n=1)$ was 
computed. Surely, this review could reinforce the conclusions of the present study.

In the future, in a prospective study setting, it would be important to evaluate the concordance between the imaging classification of EMVI made by different radiologists, given the subjective nature of scoring and the need for criteria standardization.

Regarding the different MRI-EMVI scores and the related outcomes, a mean survival rate of 68.9 months ( $\mathrm{SE}=4.2,95 \% \mathrm{Cl} 60.7-77.2)$ and a mean DFS of 73.6 months ( $\mathrm{SE}=3.8,95 \% \mathrm{Cl} 66.3$ - 81), was observed in the group of the patients with EMVI score 0 . Despite these values being higher than those found in the group of patients with EMVI score 1 or 2, the difference between the two groups isn't statistically significant $(p=.461$; $\mathrm{p}=.922$ ). In accordance with the results of Smith et al., the prognostic value of these results is as expected, since the severity of EMVI detected by MRI correlates with relapse-free survival (16). Regarding survival, to our knowledge there is no data available in literature for comparison.

According to the results that thea higher MRI-EMVI score occurs in higher T-stage, $\mathrm{T} 3$ and T4 tumors were found to be more frequent in the group of patients with EMVI in MRI (84.4. Regarding the fact that EMVI is a kind of extramural tumor extension, a direct relation between EMVI and T-stage, corresponding with EMDT, is an expected result. In fact, a strong positive correlation ( $r$ ho $=.64, p<.001$ ) was verified between EMDT and $\mathrm{EMVI}$, both detected by MRI, which means that higher EMVI score is associated with higher values of EMDT. In one of the largest series published by a University of Erlangen group, T3 tumors with EMDT $>5 \mathrm{~mm}$ were associated with a 5 years survival rate of only $54 \%$. One the other hand $\mathrm{T} 3$ tumors with $\mathrm{EMDT} \leq 5 \mathrm{~mm}$, regardless of whether lymph node involvement was present, were associated with 5 years survival rate of greater than $85 \%(11,24)$. Therefore, the distinction between $\mathrm{T} 2$ and T3 stage, is not important if the T3 tumor has less than $2 \mathrm{~mm}$ spread (11). Consequently, the EMDT has clinic relevance.

As already described, for staging of rectal tumors and evaluating the prognostic factors the MERCURY Study Group protocol is recommended for large centers to obtain an optimized visualization of the rectum and its surrounding structures (25). The selection of the MRI sequences for the evaluation of EMVI and EMDT was made, whenever possible, according to the criteria included in this protocol. A protocol adherence in all technical parameters was observed in $44.4 \%$ of the cases, this was not verified in the remaining cases (55.6\%), mostly due to incomplete axial sequences or to $>4 \mathrm{~mm}$ section thickness, even so with sufficient quality for interpretation. This is an important limitation of the present study, caused by its retrospective nature.

\section{CONCLUSIONS}

In this study, it is possible to conclude that MRIEMVI score was satisfactorily concordant with histopathologic findings. According to the high specificity value and NPV which were found, it is possible to conclude that MRI-EMVI score 0 excludes, with certainty, venous involvement. If EMVI starts being used as a criteria for selection of neoadjuvant treatment, its exclusion by MRI staging may be crucial for therapy decisions and to diminish the risk of under treatment.

The recognition of MRI in the evaluation of EMVI will allow to include this prognostic factor in the multidisciplinary preoperative discussion of patients with RC.

\section{Competing interests}

The authors declare that they have no conflict of interest.

\section{Ethics approval and consent to participate}

This project was approved by Braga Hospital Ethics Committee (CESHB 123/2015) and also by Ethics Subcommittee for Life and Health Sciences. All research was performed in accordance with relevant guidelines and regulations; informed consent was obtained from all participants and/or their legal guardians.

\section{Consent for publication}

All authors listed have read and approved the submission of the manuscript and have no competing interests.

\section{Funding}

The study as no funding support.

\section{REFECRENCES}

1. International Agency for Research on Cancer [homepage na internet]. World Cancer Report 2014 (available in: http://www.iarc.fr/ en/mediacentre/pr/2014/pdfs/pr224_E.pdf)

2. Figueiras RG, Domínguez PC, Dorrego RG, Martín AV, Caamano AG. Factores pronósticos e imagen funcional delcáncer de recto. Radiología 2012; 54 (1): 45-58.

3. Popek P, Tsikitis VL. Neoadjuvant vs adjuvant pelvic radiotherapy for locally advanced rectal cancer: Which is superior? World $\mathrm{J}$ Gastroenterol. 2011;17(7): 848-854 
4. Enker WE. Total mesorectal excision - the new golden standard of surgery for rectal cancer. Ann Med. 1997;29:127-33.

5. Sauer R, Becker H, Hohenberger W, et al. Preoperative versus postoperative chemoradiotherapy for rectal cancer. N Engl J Med 2004; 351(17):1731-40.

6. Telford J, Saltzman JR, Kuntz KM. Impact of Preoperative Staging and Chemoradiation Versus Postoperative Chemoradiation on Outcome in Patients With Rectal Cancer: A Decision Analysis. Journal of the National Cancer Institute. 2004; 96(3):191-201.

7. Sebag-Montefiore D, Stephens RJ, Steele R, Monson J, Grieve R, Khanna S. Preoperative radiotherapy versus selective postoperative chemoradiotherapy in patients with rectal cancer (MRCCR07 and NCIC-CTG C016): a multicentre, randomised trial. Lancet 2009; 373: 811-20.

8. Dieguez A. Rectal cancer staging: focus on the prognostic significance of the findings described by high-resolution magnetic resonance imaging. Cancer Imaging 2013; 13(2), 277-297.

9. Smith N, Brown G. Preoperative staging of rectal cancer. Acta Oncologica 2008;47:20_31.

10. Koh D, Smith NJ, Swift RI, Brown G. The Relationship Between MR Demonstration of Extramural Venous Invasion and Nodal Disease in Rectal Cancer. Clin Med Oncol. 2008:2:267-73.

11. MERCURY Study Group. Extramural depth of tumour invasion at thin-section MR in patients with rectal cancer: results of the MERCURY Study. Radiology 2007;243:132-139.

12. MERCURY Study Group. Diagnostic accuracy of preoperative magnetic resonance imaging in predicting curative resection of rectal cancer: prospective observational study. BMJ 2006; 333:779.

13. Horn A, Dahl 0 , Morild I. Venous and neural invasion as predictors of recurrence in rectal adenocarcinoma. Dis Colon Rectum 1991 34:798-804

14. Harrison JC, Dean PJ, el-Zeky F, Vander Zwaag R. From Dukes through Jass: pathological prognostic indicators in rectal cancer. Hum Pathol 1994; 25:498-505.

15. Brown G, Radcliffe AG, Newcombe RG, Dallimore NS, Bourne MW Williams GT. Preoperative assessment of prognostic factors in rectal cancer using high-resolution magnetic resonance imaging. $\mathrm{Br}$ J Surg 2003; 90:355-364.

16. Smith NJ, Barbachano Y, Norman AR, Swift RI, Abulafi AM, Brown $G$. Prognostic significance of magnetic resonance imaging-detected extramural vascular invasion in rectal cancer. Br J Surg 2008; 95: 229-236.

17. Smith NJ, Shihab 0, Arnaout A, Swift RI, Brown G. MRI for Detection of Extramural Vascular Invasion in Rectal Cancer. American Journal of Roentgenology (AJR) 2008;191: 1517-1522.

18. SPCIR-Sociedade Portuguesa de Cirurgia. Projeto multidisciplinar sobre cancro do reto em Portugal (available in: http://www.spcir. com/menu-recto-intro.html)

19. Taylor FG, Quirke P, Heald RJ, et al. Preoperative high-resolution magnetic resonance imaging can identify good prognosis stage I, II, and III rectal cancer best managed by surgery alone: a prospective, multicenter, European study. Ann Surg. 2011;253:711-719.

20. Kapiteijn E, Marijnen CA, Nagtegaal ID, et al: Preoperative radiotherapy combined with total mesorectal excision for resectable rectal cancer. N Engl J Med. 2001;345:638-646.

21. Brown G, Davies S, Williams GT, Bourne MW, Newcombe RG, Radcliffe AG, Blethyn J, Dallimore NS, Rees BI, Phillips CJ. Effectiveness of preoperative staging in rectal cancer: digital rectal examination, endoluminal ultrasound or magnetic resonance imaging? Br J Cancer 2010; 91(1):23-29.

22. Talbot IC, Ritchie S, Leighton MH, Hughes AO, Bussey HJ, Morson $\mathrm{BC}$. The clinical significance of invasion of veins in cancer of the rectum. Br J Surg 1980; 67:439-442.

23. Messenger DE, Driman DK, Kirsch R. Developments in the assessment of venous invasion in colorectal cancer: implications for future practice and patient outcome. Hum Pathol 2012; 43: 965-973.

24. Merkel S, Mansmann U, Siassi M, Papadopoulos T, Hohenberger W, Hermanek P. The prognostic inhomogeneity in pT3 rectal carcinomas. Int J Colorectal Dis 2001;16:298-304.

25. Glimelius B, Pahlman L, Cervantes A. Rectal cancer: ESMO clinical practice guidelines for diagnosis, treatment and follow-up. Ann Oncol 2010; 2:v82-v86. 